\title{
A comparative study of failure criteria applied to composite materials
}

\author{
E.H. Irhirane ${ }^{1}$, J. Echaabi ${ }^{1, a}$, M. Hattabi ${ }^{1}$, M. Aboussaleh ${ }^{2}$ and A. Saouab ${ }^{3}$ \\ 1 Équipe de Recherche Appliquée sur les Polymères, Département de Génie Mécanique, ENSEM, \\ Université Hassan II Aïn Chok, BP 8118, Oasis, Casablanca, Morocco \\ 2 Laboratoire de Mécanique, Département de Génie mécanique, ENSAM, Université Moulay Ismail, Présidence, Marjane 2, \\ BP 298 Mekness, Morocco \\ ${ }^{3}$ Laboratoire d'Ondes et Milieux Complexes, FRE 3102 CNRS, 53 rue Prony, BP 540, 76058 Le Havre Cedex, France
}

Received 25 December 2007, accepted 1st April 2008

\begin{abstract}
This article presents an analytical and numerical investigation of the failure loads, successive failures and failure modes of laminated beams. Two simulations were developed to model various composites behaviours under transverse static loading. Progressive failure analysis has been carried out in linear and elastic range. For the two simulations, the first order shear deformation theory with shear correction factor and the finite element method have been used respectively. The main objective of this paper is to evaluate the overall damage and successive failure for different laminates obtained by failure criteria and then to conduct a comparative study of the commonly used failure criteria. Various failure criteria have been studied to predict the load, when the weakest lamina fails under flexural bending test. After the failure of the weakest ply, the stiffness is reduced to account for fibre or matrix failures. The failure criteria are tested with various stiffness reduction models. Comparisons are made between the failure loads, successive failures, failure modes, macroscopic curves and the total behaviour curves obtained by the various failure criteria used.
\end{abstract}

Key words: First ply failure; failure load; successive failur; failure criteria; stiffness reduction.

\section{Introduction}

Currently, composite materials are largely used in a majority of the industrial sectors in a current way. However, a substantial effort is still needed for their optimal use. Dimensioning tools must be effective, reliable and precise to improve design optimisation. An alternative to facilitate the analysis of failure behaviour of composite materials consists in using failure criteria. These criteria are largely used in the commercials finite elements codes for the dimensioning of the composites materials. However, the problem of the choice of the appropriate criterion to predict failure has not received enough interest. Indeed, a great number of formalisms exist [1-3] and new or simply modified ones are still published [4-6]. The choice among these formalisms is difficult because of their diversity and because they must be validated by biaxial tests which are both expensive and difficult to realize [3-7].

In this work, we propose to study three types of formalisms representative of the great families of failure criteria; 1 . non interactive failure criteria such as the maximum stress and the maximum strain; 2 . interactive failure criteria such as Tsai Hill and the well now Tsai Wu cri-

\footnotetext{
${ }^{a}$ Corresponding author: j.echaabi@ensem-uh2c.ac.ma
}

teria; 3. finally, failure criteria which take into account physical considerations such as Hashin and Hart-Smith criteria.

In this study, we use the laminates theory with transverse shearing taking account of the coefficients of shearing and the finite element method. An analytical and a numerical simulation were developed to model the progression of damage. In general, after the first failure and for some configurations, the laminate can still support efforts which depend on its nature and the type of the stress in action. Several authors have developed approaches to relate the state of damage to the principal characteristics of the material $[8,9]$. Thereafter, the total discount and the limited discount methods for the reduction of the coefficients of the matrix of rigidity are used. For certain configurations, the three points bending test makes it possible to highlight how a material is degraded gradually until the final failure $[10,11]$. For this effect, we carry out the simulation of three points bending test on standardized specimens [12]. The failure loads, the successive failures, the failure modes, the progression of the damage, the effects of the geometrical parameters of the specimens and of the stacking sequences were studied. 


\section{Theoretical failure analysis}

\subsection{Constitutives equations}

The formalism used in this work is based on the laminates law with transverse shearing and take account of the coefficients of correction. The constitutive equation is written in the following form:

$$
\left[\begin{array}{l}
N_{i j} \\
M_{i j} \\
Q_{y} \\
Q_{x}
\end{array}\right]=\left[\begin{array}{llll}
A_{i j} & B_{i j} & 0 & 0 \\
B_{i j} & D_{i j} & 0 & 0 \\
0 & 0 & F_{44} & F_{45} \\
0 & 0 & F_{45} & F_{55}
\end{array}\right]\left[\begin{array}{c}
\varepsilon_{i j}^{0} \\
\kappa_{i j} \\
\gamma_{y z}^{0} \\
\gamma_{x z}^{0}
\end{array}\right]
$$

$N_{i j}, Q_{x}$ and $Q_{y}$ are the resulting forces and $M_{i j}$ are the resulting moments. $A_{i j}, B_{i j}, D_{i j}(i, j=1,2,6)$ and $F_{i j}$ $(i, j=4,5)$ are the matrices of rigidity of the laminate, with:

$$
\left\{\begin{array}{l}
A_{i j}=\sum_{k=1}^{n}\left(Q_{i j}^{\prime}\right)_{k}\left(h_{k}-h_{k-1}\right) ; \\
B_{i j}=\frac{1}{2} \sum_{k=1}^{n}\left(Q_{i j}^{\prime}\right)_{k}\left(h_{k}^{2}-h_{k-1}^{2}\right) ; \\
D_{i j}=\frac{1}{3} \sum_{k=1}^{n}\left(Q_{i j}^{\prime}\right)_{k}\left(h_{k}^{3}-h_{k-1}^{3}\right) ; \\
F_{i j}=\sum_{k=1}^{n} K_{i} K_{j}\left(C_{i j}^{\prime}\right)_{k}\left(h_{k}-h_{k-1}\right)
\end{array}\right.
$$

where $Q_{i j}^{\prime}$ and $C_{i j}^{\prime}$ are the expressions for the reduced stiffness apart from its principal axes, $h_{k}$ and $h_{k-1}$ are, respectively, the distances of the higher and lower interfaces of the layer $k$ from the center of the laminate (Fig. 1) and $K_{i}$ is the correction coefficient of shearing.

\subsection{Failure criteria}

The failure of composite materials can be treated from the microscopic or macroscopic point of view. The analysis of composites at the microscopic level is difficult and complex to model. The macroscopic behaviour modelling begins from the data of load displacement of a specimen subjected to a test. The majority of the failure criteria are phenomenological, in the sense that they are not deduced from a micromechanical analysis. Consequently, they can be accepted or rejected only by comparison with experimental data. The failure criteria applied to composite materials are difficults to formulate because these later have a structural and material complexity. A simple criterion cannot model with precision the failure of composites. It is thus necessary to increase his complexity but at the same time it becomes more difficult to implement. In practice, each criterion is used for particular experimental results. Until date, there is no general and systematic approach to describe failure of composite materials.

The validity of the failure criteria is discussed in general according to various aspects: linear and nonlinear effects, failure modes and their interaction, the effect of shearing and the type of the laminate etc. More researches are necessary in particular on the failure modes and their

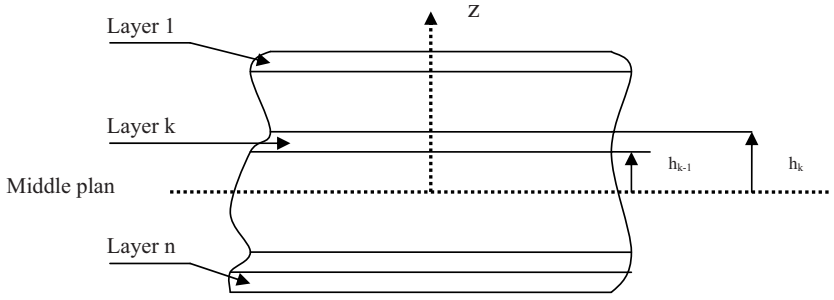

Fig. 1. Laminate geometry.

interaction. One of the objectives of our work consists in validating the reliability and the precision of the most answered failure criteria;

1. Non interactive criteria such as the maximum stresses and the maximum strains ones whose mechanisms of longitudinal, of transverse or of shearing occur independently. The failure stresses and the failure modes are predicted. But only the linear behaviour is described. These criteria over-estimate the failure stresses in the corners of the rectangle (failure envelope).

2. Interactive criteria such as Tsai Hill and Tsai Wu ones: the failure stresses are predicted but the failure modes cannot be predicted. The problem in the application of Tsai $\mathrm{Wu}$ criterion is the determination of the coefficients of interaction. Several methods were proposed to evaluate these coefficients. In our case, the following equation:

$$
F_{i j}=-\frac{1}{2} \sqrt{F_{i i} F_{j j}}
$$

is used to calculate the coefficients of interaction. These criteria cannot correctly describe the complexity of the failure of composite materials. However, they can be used in practice, when their precision is satisfactory and that no other method is valid.

3. A number of criticisms [3] were formulated by Hashin [13] and recently by Hart-Smith [14] about the limitations and the problems encountered when using the interactive failure criteria. Hashin has developed for transversally isotropic unidirectional laminates a quadratic polynomial criterion which takes into account physical considerations. Four distinct failure modes are considered separately. Two important items were discussed by this criterion, the incorporation of the failure modes by proposing for each mode an equation and the possibility of interaction of the stress. Until now, the matrix failure modes remains difficult to model.

The method of Hart-Smith is based on the criterion of the maximum shear stress in the space of the strains. The originality of the work of Hart-Smith is not in the choice of the criterion of maximum shear stress, which was used previously nor in its criticism of the traditional criteria which had been announced by some authors, but rather in the incorporation of the physical considerations. The criterion is derived for strong, stiff fibres embedded in a soft matrix and its validity is restricted to fibre dominated failure. To account for other failure modes, Hart-Smith 
Table 1. Mechanical characteristics of test samples.

\begin{tabular}{ccccc}
\hline$X_{\mathrm{t}} 11$ & $X_{\mathrm{C} 1}$ & $X_{\mathrm{t}} 22=X_{\mathrm{t}} 33$ & $X_{\mathrm{c}} 22=X_{\mathrm{c}} 33$ & $X 12=X 13$ \\
\hline 2.647 & 1.723 & 0.0514 & 0.222 & 0.0861 \\
\hline
\end{tabular}

Table 2. Specimen characteristics and specifications.

\begin{tabular}{cccccccc}
\hline $\begin{array}{c}\text { Laminates } \\
\text { Type }\end{array}$ & $\begin{array}{c}\text { Layup } \\
(\text { degrees })\end{array}$ & $\begin{array}{c}\text { \# of } \\
\text { Plies }\end{array}$ & $\begin{array}{c}\text { Length } L \\
(\mathrm{~mm})\end{array}$ & $\begin{array}{c}\text { Span } l \\
(\mathrm{~mm})\end{array}$ & $\begin{array}{c}\text { Width } b \\
(\mathrm{~mm})\end{array}$ & $\begin{array}{c}\text { Thickness } h \\
(\mathrm{~mm})\end{array}$ & $\begin{array}{c}\text { Ratio } \\
l / h\end{array}$ \\
\hline $\mathrm{A}$ & {$\left[90_{8} / 0_{8}\right]_{\mathrm{s}}$} & 32 & 200 & 152.5 & 25 & 4.736 & 32 \\
$\mathrm{~B}$ & {$\left[08 / 90_{8}\right]_{\mathrm{s}}$} & 32 & 200 & 152.5 & 25 & 4.736 & 32 \\
$\mathrm{C}$ & {$[0 / 90]_{8 \mathrm{~s}}$} & 32 & 200 & 152.5 & 25 & 4.485 & 34 \\
$\mathrm{D}$ & {$[45 / 0 /-45]_{5 \mathrm{~s}}$} & 30 & 200 & 152.5 & 25 & 4.236 & 36 \\
$\mathrm{E}$ & {$[45 /-45 / 90 / 0]_{3 \mathrm{~s}}$} & 24 & 150 & 115.0 & 10 & 3.600 & 32 \\
$\mathrm{~F}$ & {$[45 /-45 / 90 / 0]_{3 \mathrm{~s}}$} & 24 & 115 & 57.0 & 10 & 3.600 & 16 \\
\hline
\end{tabular}

Table 3. Successive failures results of laminates (A, B, C) with the total discount method.

\begin{tabular}{|c|c|c|c|}
\hline Criterion tested & Sample A $\left[90_{8} / 0_{8}\right]_{\mathrm{s}}$ & Sample B $\left[0_{8} / 90_{8}\right]_{\mathrm{s}}$ & Sample C $[0 / 90]_{8 \mathrm{~s}}$ \\
\hline Tsai Hill & $1-2-3-4-5-6-7-8$ & 9-10-11-32-12-13-31-14 & $\underline{2-4-6-8-10}-1-32-30$ \\
\hline Tsai Wu & $\underline{1-2-3-4-5-6-7-8}$ & $\underline{9-10-11-12-3}$ & $\underline{2-4-6-8-10-1}$ \\
\hline Maximum strain & 1-2-3-4-5-6-7-8 & 9-10-11-32-12-13-31-14 & $2-4-6-8-10-32-12-30$ \\
\hline Maximum stress & $\underline{1-2-3-4-5-6-7-8}$ & $\underline{9-10-11-32-12-13-31-14}$ & $\underline{2-4-6-8-10-32-12-1}$ \\
\hline Hashin & 1-2-3-4-5-6-7-8 & 9-10-11-32-12-13-31-14 & 2-4-6-8-10-32-12-1 \\
\hline Harth Smith & 1-2-3-4-5-6-7-8 & $1-32-31-2-3-30-29-4$ & $32-1-31-2-30-3-29-4$ \\
\hline
\end{tabular}

$\underline{\mathrm{xxx}}$ is the same successive failures predicted by the failure criteria.

truncated the failure envelopes. A comparison with the experimental results is still necessary to conclude on the reliability and the general information of this approach.

\section{Analytical results and discussion}

The present study uses a graphite epoxy AS1/3052 specimens. The failure stresses parameters are presented in Table 1. Six rectangular specimens are used to study the influence of the stacking sequence and the effects of the geometrical characteristics of specimens on the successive failure. Table 2 illustrates the geometrical characteristics and the stacking sequences of the test-specimens. The dimensions are those recommended by ASTM specifications. In the first part of this work, an analytical study of successive failure and failure modes is presented. The theory of laminates with transverse shearing taking into account the coefficients of shearing correction is used. A finite element method is used in the second part.

The objective is to evaluate the results obtained by the failure criteria used in the various specimens and to elaborate a comparative study between these criteria.

The evaluation of the stress and the strain fields makes it possible to determine the first time when failure is reached; the failure criterion is satisfied. The program elaborated detects the failure position and the corresponding ply. Then the corresponding coefficients in the matrix of stiffness of the layer are then modified. A new analysis is made with a modified stiffness matrix [ABCD].
The analytical results obtained by the six failure criteria used are represented in the form of tables. The latter clearly shows the failure succession, the failure loads, displacements and the position of each failure. The study is made on the first eight successive failures with two models of stiffness reduction: 1 . total discount method; 2 . limited discount method.

1. In the total discount method, the stiffness and strength of a failed ply are reduced to zero, although the ply is still physically present. The analysis of the results obtained with this approach enables us to make the following conclusions.

For all the specimens, the results of successive failures are presented in Tables 3 and 4. For type A specimens, all the failure criteria give the same predictions (successive failures, load and displacement at failure). All the failures observed in the 32 plies show that, before the failure occurred in the $0^{\circ}$ ply, no difference between the predictions of the five criteria is observed. A light divergence was noted on the level of the breaking loads of the $0^{\circ}$ ply (Tab. 5). After this macroscopic failure, a weak difference was noticed between the various predictions.

For type B specimens, the failure succession is identical for the maximum strain, maximum stress, Tsai Hill and Hashin criteria. A small difference between their breaking loads was observed. The first three failures obtained by Tsai $\mathrm{Wu}$ are identical to those predicted by the others criteria. Hart-Smith always predicts alternate successive failures. However, the others criteria predict almost the same macroscopic breaking loads (Tab. 5). A disparity was noted between the predictions of the five 
Table 4. Successive failures results of laminates (D, E, F) with the total discount method.

\begin{tabular}{lccc}
\hline Criterion tested & Sample D $[45 / 0 /-45]_{5 s}$ & Sample E $[45 /-45 / 90 / 0]_{3 \mathrm{~s}}$ & Sample F $[45 /-45 / 90 / 0]_{3 \mathrm{~s}}$ \\
\hline Tsai Hill & $29-26-1-2-23-4-25$ & $3-1-7-2-21-5-6-22$ & $3-1-7-2-21-5-6-22$ \\
Tsai Wu & $29-26-2-23-1-5-20-4$ & $3-1-7-2-5-21-6-4$ & $3-1-7-2-5-21-6-4$ \\
Maximum strain & $29-1-30-3-26-4-6-28$ & $3-7-21-1-2-5-22-17$ & $3-7-21-1-2-5-22-17$ \\
Maximum stress & $29-1-28-3-27-4-25-6$ & $3-7-24-21-1-2-23-5$ & $3-7-24-21-1-2-23-5$ \\
Hashin & $29-1-26-2-4-3-23-5$ & $3-24-7-23-1-2-5-20$ & $3-24-7-23-1-2-5-20$ \\
Harth Smith & $29-2-26-5-8-23-20-11$ & $3-22-4-21-7-18-8-17$ & $3-22-4-21-7-18-8-17$ \\
\hline
\end{tabular}

Table 5. Failure loads for samples A and B.

\begin{tabular}{lcccccc}
\hline & \multicolumn{2}{c}{ Sample A $\left[90_{8} / 0_{8}\right]_{s}$} & & \multicolumn{2}{c}{ Sample B $\left[0_{8} / 90_{8}\right]_{s}$} \\
\hline & FPF load & $\begin{array}{c}\text { M.FPF load } \\
\text { Total dicount }\end{array}$ & $\begin{array}{c}\text { M.FPF load } \\
\text { Marix unchanged }\end{array}$ & FPF load & $\begin{array}{c}\text { M.FPF load } \\
\text { Total dicount }\end{array}$ & $\begin{array}{c}\text { M.FPF load } \\
\text { Marix unchanged }\end{array}$ \\
\hline Tsai Hill & $278(1)^{*}$ & $1102(9)$ & - & $2506(9)$ & $3826(32)$ & - \\
Tsai Wu & $278(1)$ & $1165(24)$ & - & $2454(9)$ & $4764(32)$ & - \\
Maximum strain & $278(1)$ & $1165(24)$ & $1911(24)$ & $2414(9)$ & $3833(32)$ & $3887(32)$ \\
Maximum stress & $278(1)$ & $1160(24)$ & $1894(24)$ & $2510(9)$ & $3819(32)$ & $3866(32)$ \\
Hashin & $278(1)$ & $1160(24)$ & $1894(24)$ & $2510(9)$ & $3819(32)$ & $3866(32)$ \\
Harth Smith & $278(1)$ & $1220(24)$ & -3031 & $(1)$ & $3031(1)$ & - \\
\hline
\end{tabular}

FPF load is the first ply failure load expressed in N,

M.FPF load is the macroscopic first ply failure load expressed in N,

()* Failure location, given by ply number,

Note: ply number starts from bottom to top.

Table 6. Failure loads for samples C and D.

\begin{tabular}{lcccccc}
\hline & \multicolumn{3}{c}{ Sample C[0/90 $]_{8 \mathrm{~s}}$} & \multicolumn{2}{c}{ Sample D $[45 / 0 /-45]_{5 \mathrm{~s}}$} \\
\cline { 2 - 7 } & $\begin{array}{c}\text { FPF } \\
\text { load }\end{array}$ & $\begin{array}{c}\text { M.FPF load } \\
\text { Total dicount }\end{array}$ & $\begin{array}{c}\text { M.FPF load } \\
\text { Marix unchanged }\end{array}$ & $\begin{array}{c}\text { FPF } \\
\text { load }\end{array}$ & $\begin{array}{c}\text { M.FPF load } \\
\text { Total dicount }\end{array}$ & $\begin{array}{c}\text { M.FPF load } \\
\text { Marix unchanged }\end{array}$ \\
\hline Tsai Hill & $753(2)$ & $2147(1)$ & - & $1095(29)$ & $1282(2)$ & - \\
Tsai Wu & $749(2)$ & $2639(1)$ & - & $861(29)$ & $1382(2)$ & - \\
Maximum strain & $746(2)$ & $2191(32)$ & $2323(32)$ & $759(29)$ & $968(2)$ & $2563(2)$ \\
Maximum stress & $753(2)$ & $2177(32)$ & $2304(32)$ & $963(29)$ & $1355(2)$ & $2591(2)$ \\
Hashin & $753(2)$ & $2177(32)$ & $2304(32)$ & $1459(29)$ & $1424(2)$ & $2591(2)$ \\
Harth Smith & $1812(32)$ & $1812(32)$ & - & $1034(29)$ & $1034(29)$ & - \\
\hline
\end{tabular}

failure criteria after the failure of the ply $0^{\circ}$. The maximum stress and Hashin criteria practically lead to identical results.

For type $\mathrm{C}$ specimens, a good correlation for the successive failure is observed with the maximum stress, maximum strain and Hashin criteria, but with different breaking loads (Tab. 6).

For type D specimens, it appears that there is a notable difference between the predictions of the six failure criteria. For the laminates of the type $\mathrm{D}$ (mixture of $0^{\circ}$ and $45^{\circ}$ ), It is impossible to distinguish between the results obtained for the six failure criteria. This type of laminates tends to have a uniform distribution of rigidity along the thickness compared to types A and B laminates. The criterion of Hashin predicts breaking loads larger than those obtained by the maximum stress criterion (Tab. 6).

For type $\mathrm{E}$ and $\mathrm{F}$ specimens, all the failure criteria predict the first failure in the same ply (ply 3) with a small differences between the values of the breaking loads (Tab. 7). Confrontation between the successive failures obtained by the six criteria shows that there is a great disparity between them. As a conclusion of this part, a very small variation in the results of the specimen $A, B$ and $\mathrm{C}$ was observed. The maximum stress and Hashin criteria lead practically to identical predictions for the first three specimens.

For the specimens D, E and F, it is difficult to distinguish between the predictions obtained by the six failure criteria.

2. The limited discount approach considers the stiffness reduction dependent on the failure mode in action. Only failure criteria allowing the prediction of the failure modes can be used. For that, only the maximum stress, the maximum strain and the Hashin criteria are used. In this method of stiffness reduction, the failure succession, the breaking loads and the ply where failure occurred are predicted but also the failure modes. If the rupture occurs 


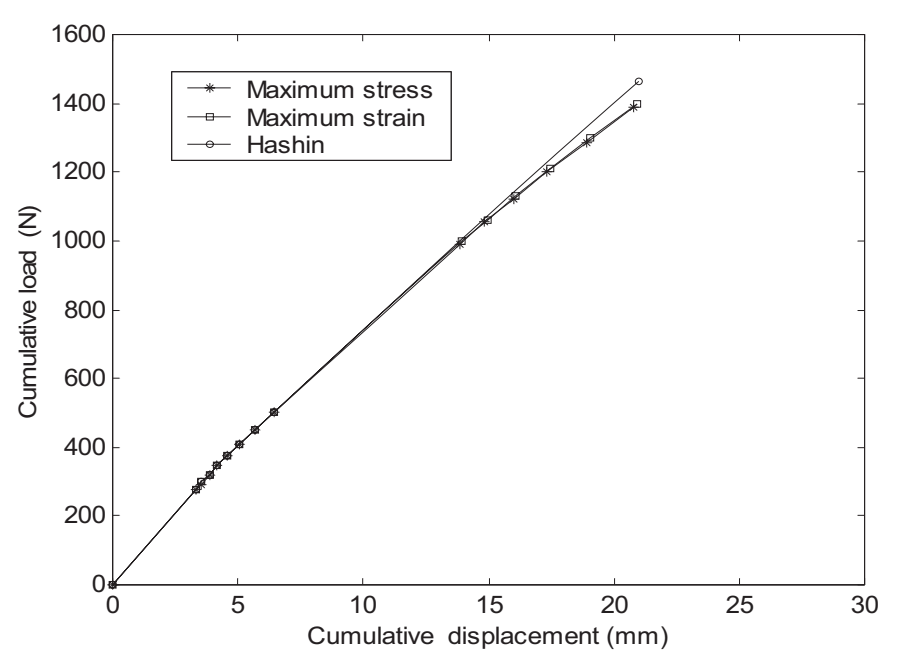

Fig. 2. Cumulative macroscopic curves of the test sample A with the limited discount method (matrix stiffness reduction).

by fibre, the total discount approach is applied and if the rupture occurs by matrix cracking, two cases were treated; 1. in the first case, all the components of the matrix of rigidity of the resin are reduced to zero; 2 . in the second one, the matrix of rigidity remains unchanged. The analysis of the results obtained for the two cases of reduction of the matrix of rigidity, makes it possible to draw the following remarks:

A good correlation between the successive failure and the failure modes is obtained for the specimens A, B and $\mathrm{C}$.

The breaking loads of the specimen A are practically identical for the three failure criteria;

The breaking loads of the specimen $\mathrm{B}$ and $\mathrm{C}$ are of the same value for the maximum stress and for Hashin criteria and are slightly larger than those predicted by the maximum stress criterion.

It is impossible to distinguish between the successive failures obtained with the specimens D, E and F.

According to position of failure, one notes that there is a uniform distribution of rigidity along the thickness for the specimens D, E and F. For the same specimens, the Hashin and the maximum stress criteria predict the same macroscopic breaking load in the second case of stiffness reduction.

The macroscopic breaking loads (failure of $0^{\circ}$ ply) obtained for the second case of stiffness reduction are slightly larger than those predicted with the first case. The approach of the limited discount of rigidity does not have a great influence on the successive failures, but it increase the breaking loads.

The total behaviour can be regarded as parameter of comparison between the failure criteria. The total or cumulative curve is the superposition of the curves of various loadings after each failure. Figures 2 to 5 present the cumulative curves obtained from the various models of stiffness reduction presented earlier. The representation is limited to specimens A and E. Each one represents a

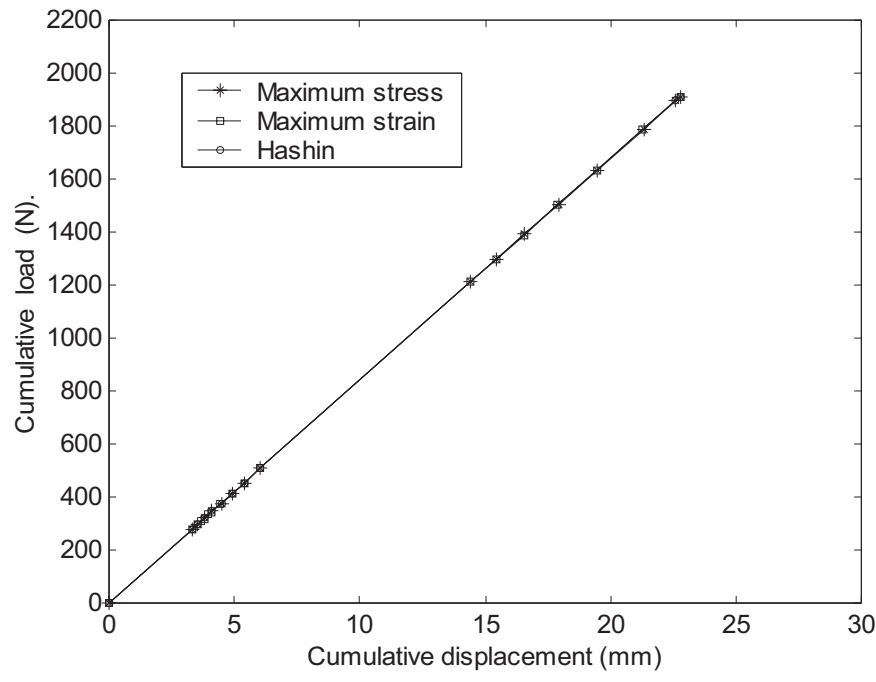

Fig. 3. Cumulative macroscopic curves of the test sample A with the limited discount method (matrix stiffness unchanged).

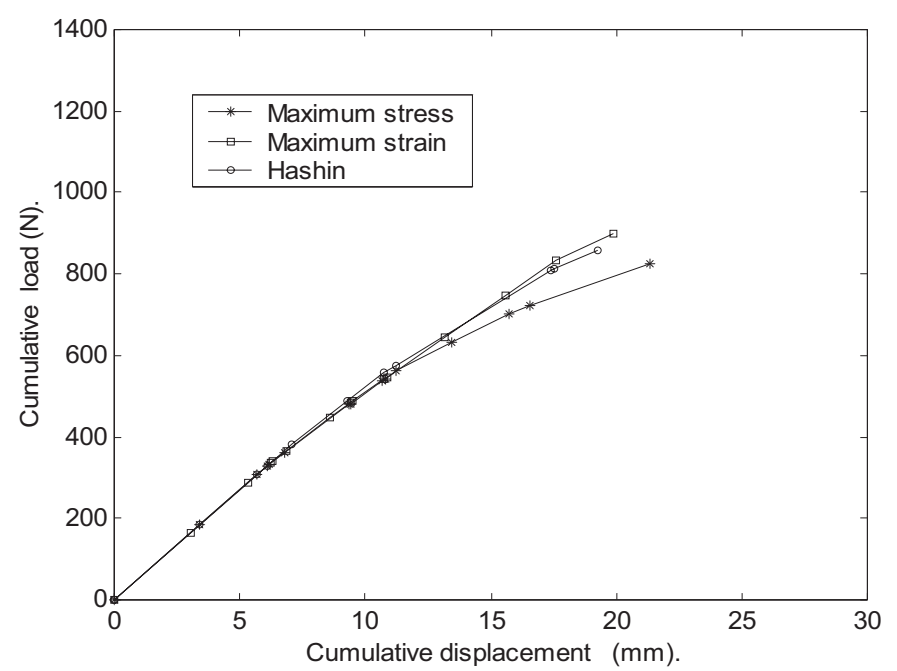

Fig. 4. Cumulative macroscopic curves of the test sample E with the limited discount method (matrix stiffness reduction).

family of the studied specimens. These curves show the maximum loads at the final failure of the laminate. This cumulative charge is the sum of the increments of the forces necessary to produce a failure in each layer. It appears that the cumulative curves obtained with the second case of stiffness reduction are almost confused. What shows that the failure criteria used predict the same total behaviour and consequently the same flexural rigidity.

\section{Numerical results and discussion}

A data-processing program based on the finite element method was elaborated within our laboratory to simulate the deflection of the specimens. The element used is a solid with eight nodes. The classical formulation in terms 
Table 7. Failure loads for samples E and F.

\begin{tabular}{lcccccc}
\hline & \multicolumn{3}{c}{ Sample E[45/-45/90/0 $]_{3 \mathrm{~s}}$} & \multicolumn{3}{c}{ Sample F $[45 /-45 / 90 / 0]_{3 \mathrm{~s}}$} \\
\cline { 2 - 7 } & $\begin{array}{c}\text { FPF } \\
\text { load }\end{array}$ & $\begin{array}{c}\text { M.FPF load } \\
\text { Total dicount }\end{array}$ & $\begin{array}{c}\text { M.FPF load } \\
\text { Marix unchanged }\end{array}$ & $\begin{array}{c}\text { FPF } \\
\text { load }\end{array}$ & $\begin{array}{c}\text { M.FPF load } \\
\text { Total dicount }\end{array}$ & $\begin{array}{c}\text { M.FPF load } \\
\text { Marix unchanged }\end{array}$ \\
\hline Tsai Hill & $183(3)$ & $368(21)$ & - & $371(3)$ & $744(21)$ & - \\
Tsai Wu & $172(3)$ & $302(21)$ & - & $348(3)$ & $609(21)$ & $1012(21)$ \\
Maximum strain & $165(3)$ & $263(21)$ & $501(21)$ & $333(3)$ & $531(21)$ & $1012(21)$ \\
Maximum stress & $185(3)$ & $330(21)$ & $615(21)$ & $374(3)$ & $666(21)$ & $1241(21)$ \\
Hashin & $185(3)$ & $407(21)$ & $615(21)$ & $374(3)$ & $823(21)$ & $1241(21)$ \\
Harth Smith & $206(3)$ & $345(4)$ & - & $834(3)$ & $714(4)$ & - \\
\hline
\end{tabular}

Table 8. Successive failures results obtained by numercical analysis.

\begin{tabular}{lccc}
\hline Sample & Tsai Wu & Maximum stress & Maximum strain \\
\hline $\mathrm{A}\left[90_{8} / 0_{8}\right]_{\mathrm{s}}$ & $32-31-30-29-28-27-26-25$ & $32-31-30-29-28-27-26-25$ & $32-31-30-29-28-27-26-25$ \\
$\mathrm{~B}\left[0_{8} / 90_{8}\right]_{\mathrm{s}}$ & $24-23-22-21-20-19-18-17$ & $24-23-22-21-20-19-18-17$ & $24-32-23-31-22-30-21-29$ \\
$\mathrm{C}[0 / 90]_{8 \mathrm{~s}}$ & $31-29-27-25-23-21-2-19$ & $31-29-27-25-23-2-21-4$ & $31-29-27-25-2-23-4-21$ \\
$\mathrm{D}[45 / 0 /-45]_{5 \mathrm{~s}}$ & $30-1-3-2-28-27-25-4$ & $30-28-27-25-1-3-2-24$ & $1-3-2-4-5-6-7-30$ \\
$\mathrm{E}[45 /-45 / 90 / 0]_{3 \mathrm{~s}}$ & $24-23-22-20-19-18-16-15$ & $22-24-23-20-18-19-16-3$ & $22-24-23-18-20-3-1-19$ \\
$\mathrm{~F}[45 /-45 / 90-0]_{3 s}$ & $24-23-22-20-19-18-16-3$ & $22-24-23-20-18-19-3-16$ & $24-23-22-20-19-16-18-3$ \\
\hline
\end{tabular}

Table 9. First ply failure loads obtained by numercical analysis.

\begin{tabular}{lcccc}
\hline Sample & Tsai Wu & Maximum stress & Maximum strain & Error = Maxi-Mini/Maxi \\
\hline $\mathrm{A}\left[90_{8} / 0_{8}\right]_{\mathrm{s}}$ & 227 & 278 & 274 & $18 \%$ \\
$\mathrm{~B}\left[0_{8} / 90_{8}\right]_{\mathrm{s}}$ & 1956 & 2236 & 2553 & $23 \%$ \\
$\mathrm{C}[0 / 90]_{8 s}$ & 566 & 894 & 724 & $36 \%$ \\
$\mathrm{D}[45 / 0 /-45]_{5 \mathrm{~s}}$ & 543 & 1440 & 809 & $62 \%$ \\
$\mathrm{E}[45 /-45 / 90 / 0]_{3 \mathrm{~s}}$ & 106 & 187 & 199 & $46 \%$ \\
$\mathrm{~F}[45 /-45 / 90-0]_{3 \mathrm{~s}}$ & 234 & 414 & 409 & $43 \%$ \\
\hline
\end{tabular}

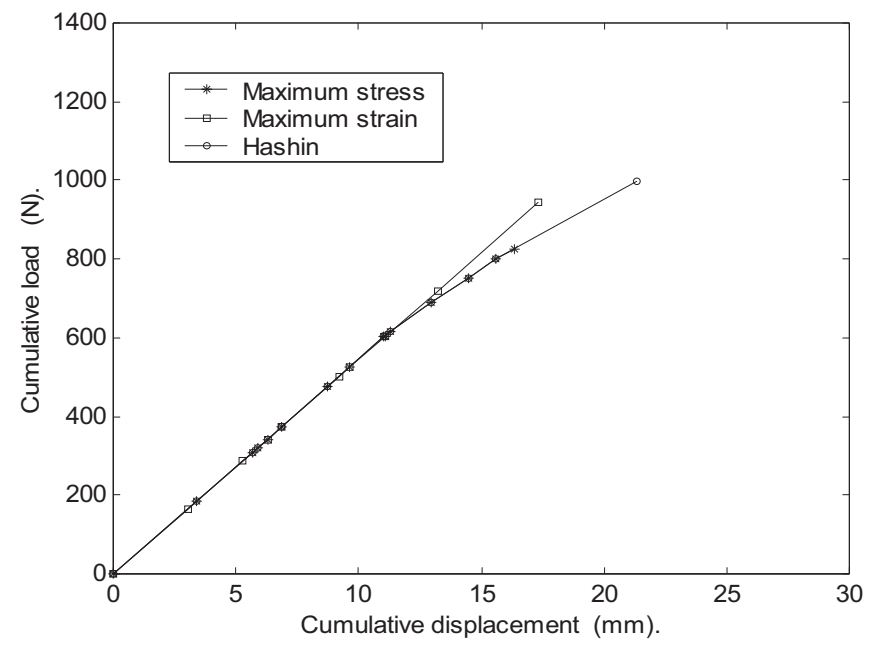

Fig. 5. Cumulative macroscopic curves of the test sample $\mathrm{E}$ with the limited discount method (matrix stiffness unchanged).

of the deformation is used. Information necessary for the calculation is the number of groups to calculate the stiffness matrix and each group consists of a set of layers.
The convergence is studied while decreasing the number of ply by group until even two elements by ply. The results mentioned in the article are gotten for one element by ply through the thickness of the specimen. A grid where the density was increased in the central part of the specimen was adopted. The evaluation of the stress and the strain fields makes it possible to determine the minimum loading for which a failure is reached in an element. When failure is reached in the most constrained element, its rigidity will be reduced. The results are saved at each stage and then make it possible to follow the evolution of the failure behaviour. To predict the failure with this computer code, we were interested in the most used failure criteria in the commercial software: the Tsai-Wu $3 \mathrm{~d}$ criterion which, in general, under estimate the breaking load and the maximum stress and the maximum strain criteria. The results obtained by this analysis were compared with those of the analytical method.

The results for successive failures and first ply failure load obtained with the three criteria used are respectively presented in Tables 8 and 9. For specimen A, the failure criteria used give practically the same successive ruptures. An attentive look of all the results, shows that there are small divergences between the successive failure when the damage is important. The plies where failure takes place 
obtained by this analysis are not in agreement with those obtained by the analytical approach. Some difference was noted on the level of the breaking loads. The Tsai Wu criterion under-estimates slightly the load of the first rupture. For the specimen B, the succession of failure is identical between Tsai $\mathrm{Wu}$ and the maximum stress criteria. For these last two criteria, the ply $90^{\circ}$, which is in the bottom of the specimen, are broken first. On the other hand, the criterion of the maximum strain predicts a failure succession different from that of the others criteria. Indeed, the failure alternate between the plies $90^{\circ}$ and $0^{\circ}$ of the bottom of the specimen. The maximum strain criterion predicts the maximum load of the first rupture and the criterion of Tsai Wu the minimal load. For the specimen $\mathrm{C}$, the maximum strain criterion predicts a failure succession different from that predicted by the two others criteria. The first four ruptures obtained by the three criteria are practically identical. The criterion of the maximum stress predicts the maximum loads whereas Tsai Wu criterion predicts the minimal forces. For the specimen $\mathrm{D}$, the successive failure obtained by the three failure criteria are largely different. According to the positions of the ruptures, we notice that this laminate has a uniform distribution of rigidity along the thickness.

For the specimen $\mathrm{E}$ and $\mathrm{F}$, all the failure criteria predict the same successive failures but in different orders. For the two $\mathrm{l} / \mathrm{h}$ ratios, the area of failure initiation is the same. Indeed, the failure starts in bottom of the specimen and is propagated to the top. The maximum strain predicts the maximum loads for the two specimens.

\section{Conclusion}

For the symmetrical cross ply laminates $\left(0^{\circ}\right.$ and $90^{\circ}$ plies), all the failure criteria used predict almost identical successive failures and a small difference was observed between the breaking loads. In the approach of the limited discount, without reduction of the rigidity of the matrix, the macroscopic breaking loads obtained with the majority of the failure criteria are identical for angle ply laminates formed with $45^{\circ},-45^{\circ}$ and $0^{\circ}$ plies. These laminates have an uniform distribution of rigidity along the thickness. Consequently, it is difficult to distinguish between the predictions obtained with the failure criteria. In the majority of the cases, Hashin criterion predicts the largest breaking loads and the Tsai Wu one the smallest forces. The whole of the results obtained with all the approximations suggested, highlights the difficulty to predict the failure loads and the successive failures. The results depend on the criterion used.

For all the failure criteria used, the eight first successive failures occur in the same plies but the order is different. It is noticed also that the difference in the results obtained by the various criteria depends crucially on the stacking sequences. This difference is the smallest for cross ply laminates containing the $0^{\circ}$ ply in the centre of the specimen. For angle ply laminates containing $45^{\circ}$ plies, the criteria used give largely different results. Then the choice of the criterion has a substantial influence on the results of the dimensionnning. This study shows that the dimensioning of the composites by failure criteria remains an open problem. Experimental, analytical and numerical studies are still necessary to highlight the suitable criteria for each type of laminates; stacking sequences and materials.

\section{References}

1. M.N. Nahas. Technology and Research 8, 138 (1986)

2. W.W. Feng. J. Compos. Mater. 25, 88 (1991)

3. J. Echaabi, F. Trochu, R. Gauvin. Polymer Composite 17, 786 (1986)

4. Y. Hsien-Liang. J. Reinf. Plast. Compos. 22, 517 (2003)

5. S.J. DeTeresa, G.J. Larsen. J. Compos. Mater. 37, 1769 (2003)

6. C.G. Davila, P.P. Camanho, C.A. Rose. J. Compos. Mater. 39, 323 (2005)

7. J. Echaabi, F. Trochu. J. Compos. Mater. 30, 1088 (1996)

8. R. Talreja. J. Compos. Mater. 19 (1985)

9. A. EL Mahi, J.M. Berthelot, J.M. Brillaud. Comps. Struct. 30, 123 (1995)

10. P. Pal, C. Ray. J. Reinf. Plast. Compos. 21, 1505 (2002)

11. B.G. Prusty, S.K. Satsangi, C. Ray. J. Reinf. Plast. Compos. 20, 671 (2001)

12. ASTM test. American Standards of Testing and Materials (1992), p. 790 .

13. Z. Hashin. J. Appl. Mech. 47, 329 (1980)

14. L.J. Hart-Smith. Composites Science and Technology 58, 1151 (1998) 